\title{
Characterization of longitudinal acoustic waves in a fiber using an extrinsic Fabry-Perot interferometer
}

Ricardo E. Silva, Alexandre A. P. Pohl

Ricardo E. Silva, Alexandre A. P. Pohl, "Characterization of longitudinal acoustic waves in a fiber using an extrinsic Fabry-Perot interferometer," Proc. SPIE 8421, OFS2012 22nd International Conference on Optical Fiber Sensors, 84212T (17 October 2012); doi: 10.1117/12.968806

Event: OFS2012 22nd International Conference on Optical Fiber Sensor, 2012, Beijing, China 


\title{
Characterization of longitudinal acoustic waves in a fiber using an extrinsic Fabry-Perot interferometer
}

\author{
Ricardo E. Silva and Alexandre A. P. Pohl \\ Federal University of Technology - Parana, 3165 Av. Sete de Setembro, Curitiba, Brazil, 80230-901
}

\begin{abstract}
Longitudinal acoustical modes propagating in a fiber are characterized using an extrinsic Fabry-Perot interferometer. The method allows the detection of the fiber axial deflection peaks which correspond to the acoustic resonances. The method can be further used for estimating properties of acoustic excited fibers, such as acoustic frequency and wavelength, which are essential in the design of acousto-optic devices employing Bragg gratings.
\end{abstract}

Keywords: Fiber interferometer, fiber Bragg grating, longitudinal acoustic waves, acousto-optical effect.

\section{INTRODUCTION}

Acoustic waves allow the control of the spectral characteristics of fiber Bragg gratings (FBGs), which are of interest for applications in acoustic-optic modulators ${ }^{1,2}$, grating writing ${ }^{3}$, tunable reflectors ${ }^{4}$ and fiber lasers ${ }^{5}$. Figure 1 illustrates the acoustic-optic interaction in the grating. An optical mode with effective index $n_{\text {eff }}$ propagates in a non-perturbed grating, whose period is $\Lambda$, and satisfies the Bragg condition $\left(\lambda_{B}=2 n_{\text {eff }} \Lambda\right)$. The phase matching between the forward propagating optical field and the grating planes produces a narrow reflected wavelength band centered at $\lambda_{B}$ (Figure 1(a)). However, when a longitudinal acoustic wave with velocity $v_{\mathrm{a}}=f_{\mathrm{a}} \lambda_{\mathrm{a}}$ is excited along the fiber by an acousto-optical modulator (Figure 1(c)), it produces a periodic deformation that compress and stretches the grating planes. The resulting strain modulates both the modal effective index $n_{\text {eff }}$ and the grating pitch $\Lambda$, causing reflection bands to appear on both sides of the Bragg wavelength, $\lambda_{\mathrm{B}}$ (Figure 1(b)).The distance $\Delta \lambda$ between the main and the side lobe obeys the relationship ${ }^{1}$

$$
\Delta \lambda=f_{a} \frac{\lambda_{B}^{2}}{2 n_{e f f} v_{a}},
$$

where, $f_{\mathrm{a}}$ is the acoustic frequency. Once the grating reflectivity and wavelength can be controlled by the amplitude and frequency of the acoustic wave, the acousto optic effect becomes convenient for the modulation and switching of signals. However, the excitation of longitudinal acoustic waves is not a simple task, once modulators used for this purpose only work for specific resonances that depend on the transducer piezoelectric (PZT) and silica horn ${ }^{2}$. As the modulator also allows transversal excitation of the fiber, the influence of flexural acoustic modes may reduce the FBG reflectivity due to intermodal coupling. Thus, the characterization of the mechanical behavior and the resonances of the axial acoustic wave is essential to provide a more efficient operation of the acousto-optical devices. In this work, a setup based on an extrinsic Fabry-Perot interferometer (EFPI) ${ }^{6-9}$ is used to characterize the longitudinal acoustic modes propagating in a piece of fiber that makes part of an acousto-optic modulator. When this type of wave is excited, the fiber is stretched at its tip (see Figure 1(c)), making it possible to measure the acoustic frequency $f_{\mathrm{a}}$ by assessing the changes in the optical cavity, given by the distance $d$, formed by the fiber tip, which belongs to the modulator, and the sensor head (cleaved fiber length, which guides the light to a photodetector), as shown in Figure 1(d). A fraction of the light that propagates in the sensor head is partially reflected at the fiber-air interface with intensity $I_{1}$. The transmitted light is reflected $\left(I_{2}\right)$ on the axially excited fiber, whose end works as the second mirror. This results in a reflected interference pattern $\left(I_{12}\right)$ that depends basically on the distance, $d$, between the fibers, and the optical wavelength, $\lambda$, written as,

$$
I_{12}(\lambda)=I_{1}+I_{2}+2 \sqrt{I_{1} I_{2}} \cos \left(\frac{4 \pi d}{\lambda}\right) .
$$


(a)

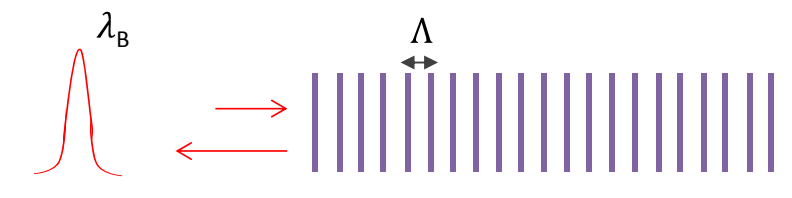

(b)

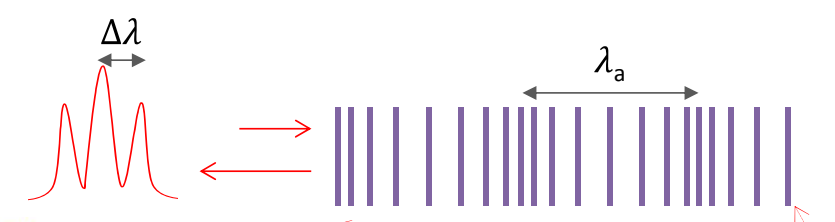

(c)

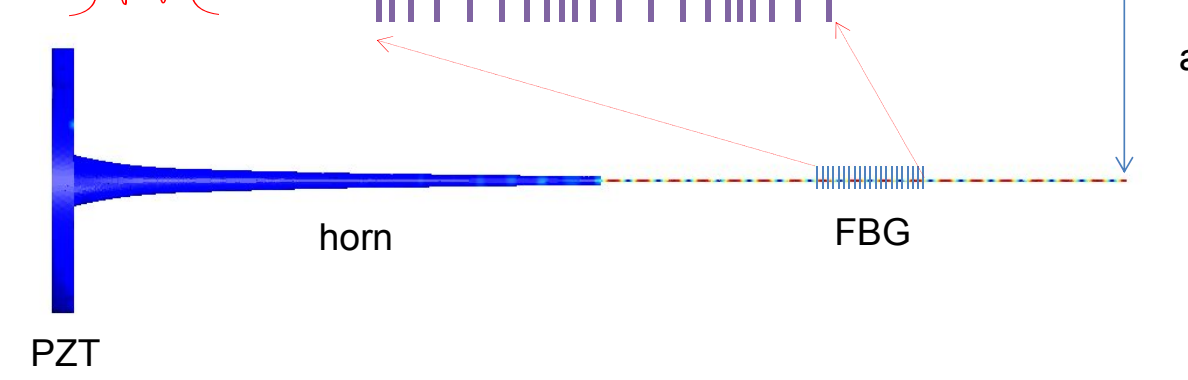

(d) optical sensor

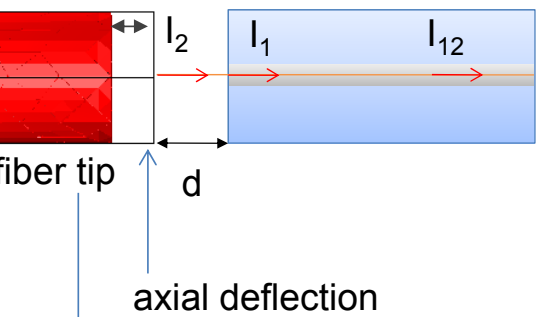

Figure 1. FBG (a) without and (b) with longitudinal acoustic wave. (c) acoustic-optic modulator and (d) optical fiber interferometer.

The optical intensity is monitored using a photodetector and an oscilloscope. Employing an appropriate method and setup based on the detection of fiber axial vibrations, the measurement of acoustic resonance frequencies is possible and demonstrated. Once the velocity $v_{\mathrm{a}}$ and the acoustic frequency $f_{\mathrm{a}}$ are known, the acoustic wavelength $\lambda_{\mathrm{a}}$ can be estimated.

\section{METHODOLOGY}

For the characterization of the acoustical longitudinal modes the experimental setup shown in Figure 2(a) is used. It consists of two optical sensor heads based on standard single mode fiber (SMF-28) and optical circulators. The sensor head 1 is used to detect the longitudinal displacement of the fiber, as shown in Figure 1(d). The sensor head 2 is positioned transversely to the fiber and is used to verify if flexural deformations are also present during the excitation. The sensor probe 2, in the form of an optical fiber ferrule, is fixed in a support mounted in a positioning table. A 50:50 coupler splits the optical power from a tunable laser between the sensor heads, with wavelength set at $\lambda=1.55 \mu \mathrm{m}$. The return signal from both sensors impinges on the photodetector, which is connected to an oscilloscope. The measurements with the sensor heads 1 and 2 are not performed simultaneously. A $5 \mathrm{~cm}$ long FBG written in a SMF has one end fixed on the tip of the silica horn and the other is free on the groove, as seen in Fig 1(c). The alignment between the modulator and the sensor heads is performed manually with the use of the positioning stages and microscopes. A piezoelectric transducer (PZT) fixed on the base of the silica horn is excited by maximum $10 \mathrm{~V}$ amplitude of an arbitrary signal generator. Varying the excitation frequency between $f=0.6-1 \mathrm{MHz}$, longitudinal acoustic waves are established, generating periodic stretching of the fiber tip. The distance variation between the probes and the excited fiber is converted into optical intensity changes, which are expressed as a voltage variation in the oscilloscope. In order to measure the resonance frequencies, the oscilloscope acquisition mode, which converts the periodic signal in its fast Fourier transform (FFT), is used. This procedure allows one to monitor the variation of the axial fiber displacement as a function of the excitation frequency. As the maximum amplitude detected by the sensor head 1 (once a flexural deformation minimum is observed by the sensor head 2) is related to the resonant frequency of the longitudinal acoustic wave, the longitudinal modes can be detected. In order to reduce the influence of environment vibration noise, the experiment is mounted on a table with a vibration absorber and the data is averaged using a 16x acquisition rate in the oscilloscope. Illuminating the grating with the broadband source and observing the reflected spectrum for the same frequency range, the influence of the longitudinal acoustic modes on the FBG spectrum, as seen in Fig. 1(b), is verified. Figure 2(b) shows the lateral view and Fig. 2(c) shows longitudinal digital microscope view just before the optimized alignment between the fiber modulator and sensor heads 1 and 2, respectively. 

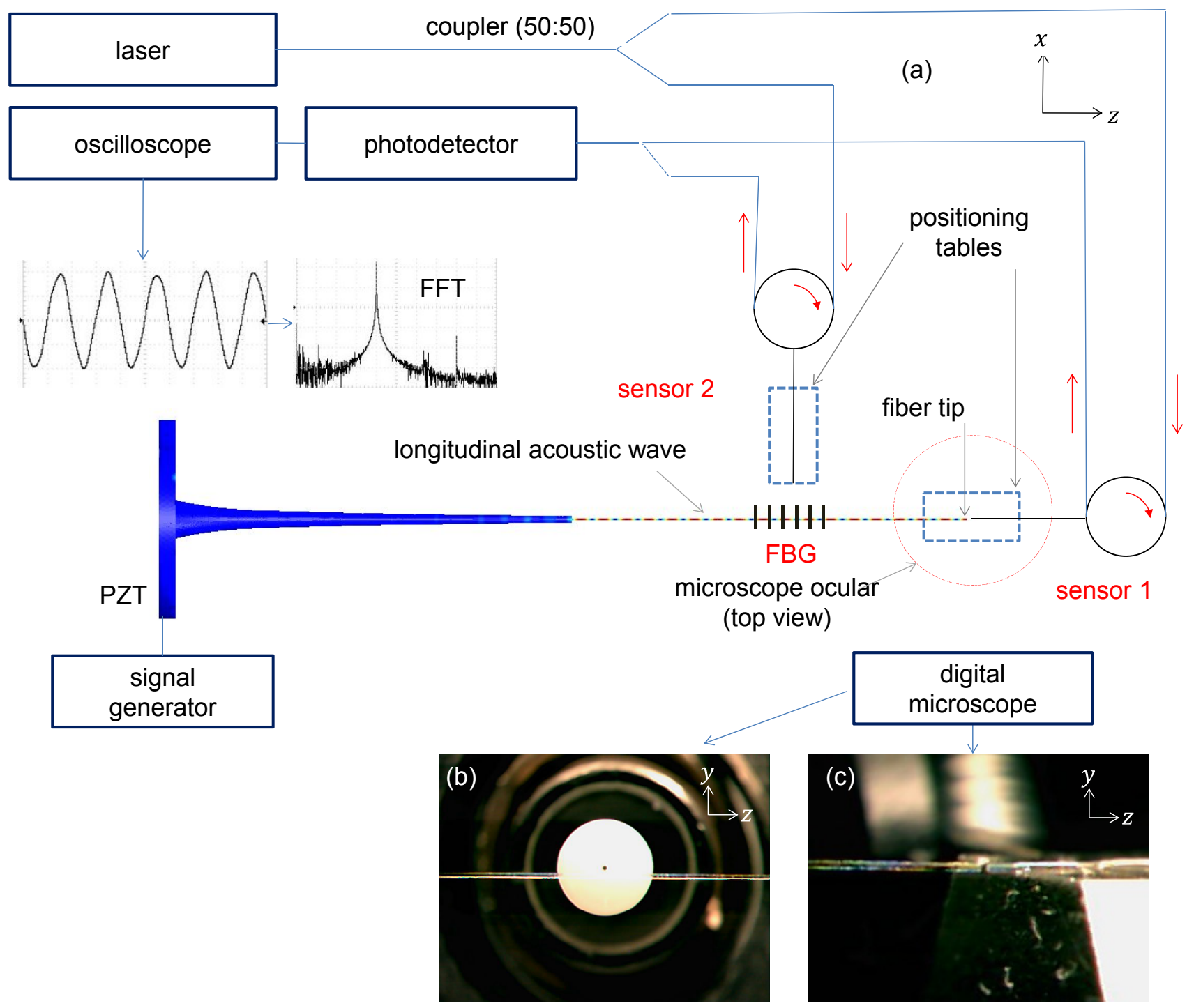

Figure 2. Experimental setup used to characterize the longitudinal acoustic waves in fiber Bragg grating.

\section{RESULTS}

Figure 3(a) shows the principal longitudinal acoustic resonances detected by the sensor head 1 over the frequency excitation range. At $f_{\mathrm{a}}=655 \mathrm{kHz}$ harmonics are observed in the oscilloscope on both sides of the fundamental resonance. This is due to the superposition of residual flexural deflections, which are remaining present in the fiber. At $f_{\mathrm{a}}=955$ and $1002 \mathrm{kHz}$ resonances show that only the axial stretching of the fiber takes place, which is linked to the excitation of a genuine longitudinal acoustic mode. Taken these measured frequencies and considering the extensional silica acoustic velocity as $v_{\mathrm{a}}=5760 \mathrm{~m} / \mathrm{s}^{1}$, the acoustic wavelength for the three axial modes are estimated as $\lambda_{\mathrm{a}}=8.79,6.03$ and $5.75 \mathrm{~mm}$, respectively. The change of the FBG spectrum, due to the presence of the longitudinal waves, is viewed in Figure 3(b). Results show the behavior of the side lobes for the three acoustic modes. The differences observed in the resonances frequencies shown in Figures 3(a) and 3(b) are due to the splicing of the fiber tip of the modulator and the fiber that belongs to the interrogator, which is necessary for assessing the FBG reflected spectrum under acousto-optic excitation. It is important to note that less than $1 \%$ difference is observed in the two cases. 

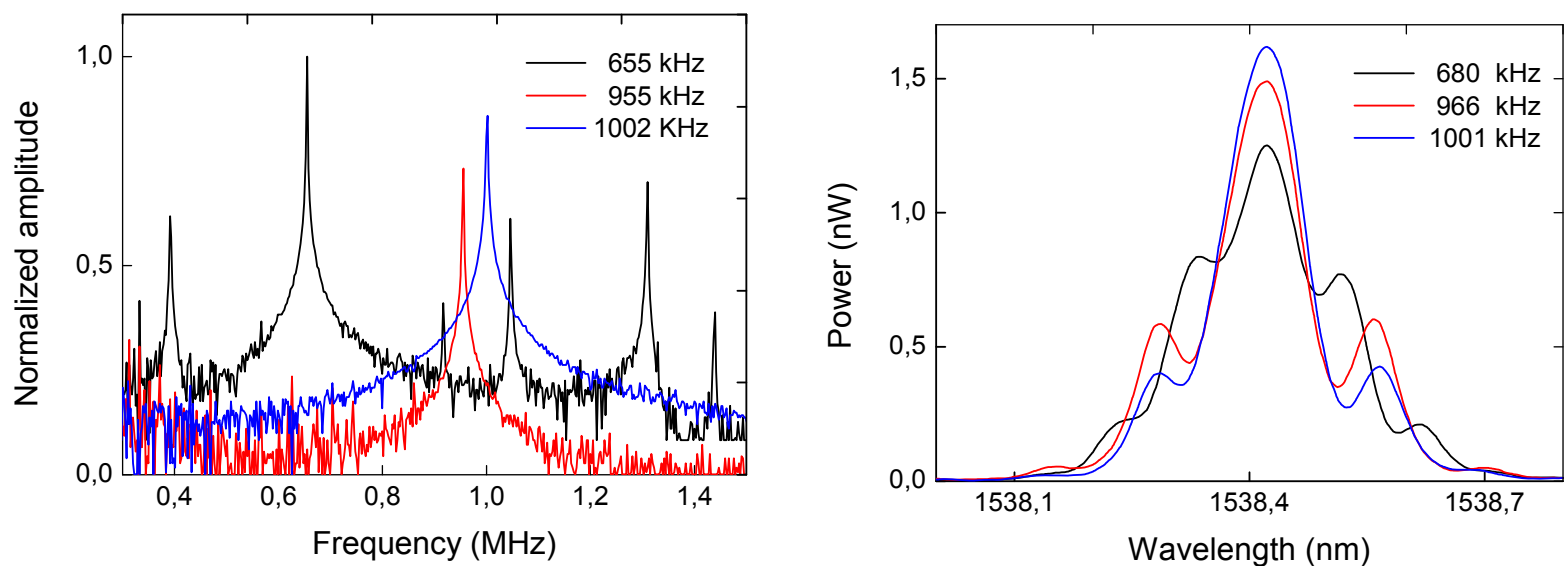

Figure 3. FFT frequency response of the longitudinal acoustic resonances and (b) and its effects on the FBG spectrum.

\section{CONCLUSION}

The characterization of longitudinal acoustic waves in fiber grating Bragg is reported. A setup based on two optical sensors allows the detection of fiber axial deflections and thus measure the frequencies of acoustic propagating modes. The transversal sensor that is used to verify that only axial modes are excited in the fiber may also be used to characterize flexural acoustic modes ${ }^{10}$. This setup can be further used for estimating the fiber acoustic properties, such as acoustic wavelength, and the displacement of the reflected band side, which are important in the control of acoustooptic devices.

\section{ACKNOWLEDGMENTS}

Ricardo E. Silva thanks the Coordenação de Aperfeiçoamento de Pessoal de Nível Superior - CAPES for the scholarships and financial support.

\section{REFERENCES}

[1] Liu, W. F., Russell P. St. J., and Dong, L., "Acousto-optic superlattice modulator using a fiber Bragg grating," Opt. Lett., 22(19), 1515-1517 (1997).

[2] Oliveira, R. A., Neves, P. T., Pereira J. T., Canning, J. and Pohl, A. A. P., "Vibration mode analysis of a silica horn fiber Bragg grating device," Opt. Commun., 283(7), 1296-1302 (2010).

[3] Oliveira, R. A., Neves Jr., P. T., Pereira J. T., Canning J. and Pohl , A. A. P., "Bragg grating writing in acoustically excited optical fiber," Appl. Phys. Letters., 97(4), 0411011-0411013 (2010).

[4] Liu, W. F., Russell, P. St. J., and Dong, L., "100\% Efficient narrow-band acoustooptic tunable reflector using fiber Bragg grating,", J. Ligh. Tech., 16(11), 2006-2009 (1998).

[5] Delgado-Pinar, M., Zalvidea, D., Diez, Perez-Millan, A., P., and Andres, M., "Q-switching of an all-fiber laser by acousto-optic modulation of a fiber Bragg grating,", Opt. Express, 14(3), 1106-1112 (2006).

[6] Gangopadhyay, T.K.; Chakravorti, S.; et al "Time-frequency analysis of multiple fringe and nonsinusoidal signals from a fiber-optic vibration sensor using an EFPI,", J. Ligh. Tech., 24(5), 2122- 2131(2006).

[7] Majumdar, A., Huang H., "Compact optical fiber white light interferometric distance sensor for arbitrary small distance measurement,", Appl. Opt. ,48(19), 3702-3708 (2009).

[8] Silva, R.E., Oliveira, R.A., Pohl, A.A.P. "Fiber Fabry-Perot interferometer sensor for measuring resonances of piezoelectric elements", Proc. SPIE 7753, OFS-21, 775319 (2011).

[9] Silva, R.E., Oliveira, R.A., Pohl, A.A.P. "Optical fiber sensor for characterization of piezoelectric transducers", Proc. IMOC 2011 (2011).

[10] Silva, R.E.,"Characterization of acoustic properties of a photonic crystal fiber acoustic-optic modulator”, UTFPR, MSc dissertation, 2011. 